\title{
Quantitative Spectral Analyses of CSPNs of Early [WC]-Type
}

\author{
L. Koesterke and W.-R. Hamann \\ Institut für Theoretische Physik und Astrophysik der Universität Potsdam
}

\section{Introduction}

Among the Central Stars of Planetary Nebulae (CSPN) there are several stars which show Wolf-Rayet-type spectra resembling those of Pop.I Wolf-Rayet (WR) stars of the carbon sequence (WC). Due to progress in computer technology and new solution techniques it became possible ten years ago to calculate models which account for the very complex physical conditions in Wolf-Rayet atmospheres (Hillier 1989, Hamann et al. 1992). These models have been successfully applied to the vast majority of Pop. I WR stars in the Galaxy and the Magellanic Clouds (Hamann et al. 1995) and, in the last three years, to an increasing sample of CSPNs of [WC] type (Koesterke \& Hamann 1996, Leuenhagen \& Hamann 1996, Leuenhagen this meeting). Here we present the analyses of ten CSPNs of early [WC] type, i.e. from [WC 2] to [WC 4].

\section{Model atmospheres and observations}

The model atmospheres are based on the so-called Standard Model for Wolf-Rayet stars, assuming homogeneity and stationarity of a spherically symmetric outflow. The consistent solution of the equations of the radiation transfer in the co-moving frame and equations of statistical equilibrium in non-LTE is achieved by the Accelerated Lambda Iteration (ALI). Up to 200 non-LTE levels are accounted for. Each model is specified by the basic parameters $T_{*}, R_{*}, \dot{M}, v_{\infty}$ and the chemical abundances $\beta_{\mathrm{He}}, \beta_{\mathrm{C}}$ and $\beta_{\mathrm{O}}$. Optical spectra of the program stars have been collected at the DSAZ (Calar Alto, Spain) and at ESO (La Silla, Chile). Three spectra have been kindly provided by $M$. Peña. IUE spectra were retrieved from the IUEDAC.

\section{Results and discussion}

While $R_{\mathrm{t}}=R_{*}\left(\frac{v_{\infty}}{2500 \mathrm{~km} / \mathrm{s}} / \frac{\dot{M}}{10^{-4} \mathrm{M}_{\odot} / \mathrm{yr}}\right)^{2 / 3}$ can be determined spectroscopically, $\dot{M}$ and $R_{*}$ scale with the adopted distance which is not known but inferred from the assumption of $L=5000 \mathrm{~L}_{\odot}$ as a typical value for CSPNs.

The determination of nitrogen remains uncertain for fainter stars for which no useful IUE observation in the short wavelength range (covering N V $1240 \AA$ ) is available. The program stars (diamonds) are shown in the figure together with late-type [WC] stars (squares) and Weak Emission Line Stars (WELS, circles). The [WCE]-stars build up a homogeneous group between 125 and $150 \mathrm{kK}$ and transformed radii between 2.0 and $6.3 \mathrm{R}_{\odot}$. It is remarkable that the stellar parameters and abundances are not correlated with the spectral subtype, although there is a weak trend to lower terminal velocities for [WC 4] stars.

Usually it is supposed that hydrogen-deficient post-AGB stars are formed within the "born-again scenario". In this scenario the stars are thrown back to the AGB when they suffer a late thermal pulse after having arrived at the White Dwarf cooling sequence. The hydrogen-rich layers are completely ejected and the bare hydrogen-deficient core becomes visible. Together with analyses of [WCL] (Leuenhagen \& Hamann 1996) and PG-1159 


\section{Central Stars}

\begin{tabular}{|c|c|c|c|c|c|c|c|c|c|c|c|}
\hline Name & HCLS & Type & $\begin{array}{c}\mathrm{m}_{\mathrm{B}} \\
\text { [mag] }\end{array}$ & $\begin{array}{c}T_{*} \\
{[\mathrm{kK}]}\end{array}$ & $\begin{array}{c}R_{\mathrm{t}} \\
{\left[\mathrm{R}_{\odot}\right]}\end{array}$ & $\begin{array}{c}R_{*} \\
{\left[\mathbf{R}_{\odot}\right]}\end{array}$ & $\begin{array}{c}\dot{M} \\
{\left[\mathrm{M}_{\odot} / \mathrm{yr}\right]}\end{array}$ & $\begin{array}{c}v_{\infty} \\
{[\mathrm{km} / \mathrm{s}]}\end{array}$ & $\beta_{\mathrm{C}}$ & $\begin{array}{l}\beta_{O} \\
\text { ass fracti }\end{array}$ & $\underset{\text { ion }}{\beta_{\mathrm{N}}}$ \\
\hline NGC5189 & TWR 13] & WC2 & 14.9 & 135.0 & 5.0 & 0.13 & -6.30 & 3000 & 0.16 & 0.08 & 0.003 \\
\hline PB 6 & & [WC 2] & 17.4 & 140.0 & 4.5 & 0.12 & -6.28 & 3000 & 0.24 & 0.14 & 0.003 \\
\hline NGC 2452 & [WR 10] & [WC2] & 17.7 & 141.0 & 4.0 & 0.12 & -6.20 & 3000 & 0.15 & 0.07 & 0 : \\
\hline NGC 2867 & [WR 12] & [WC 2] & 16.6 & 141.0 & 4.0 & 0.12 & -6.24 & 1800 & 0.25 & 0.09 & 0 : \\
\hline NGC 6905 & [WR 39] & [WC 2-3] & 16.3 & 141.0 & 3.4 & 0.12 & -6.32 & 1800 & 0.25 & 0.15 & 0 \\
\hline NGC 7026 & [WR 40] & [WC3] & 15.3 & 130.5 & 6.3 & 0.1 & -6.34 & 3500 & 0.20 & 0.10 & 0 : \\
\hline He 2-55 & & [WC 3] & 17.6 & 128.0 & 5.2 & 0.15 & -6.25 & 3000 & 0.15 & 0.06 & 0 : \\
\hline Sand 3 & & [WC 3] & $(14.2 \mathrm{~V})$ & 140.0 & 3.0 & 0.12 & -6.15 & 2200 & 0.26 & 0.12 & 0.005 \\
\hline IC 1747 & [WR 3] & [WC4] & 15.8 & 126.0 & 6.3 & 0.15 & -6.58 & 1800 & 0.25 & 0.06 & 0 \\
\hline NGC 1501 & [WR 6] & [WC4] & 15.2 & 134.5 & 3.5 & 0.1 & -6.28 & 1800 & 0.35 & 0.15 & 0 \\
\hline NGC 6369 & & [WC4] & 17.0 & 150.0 & 1.8 & 0.11 & -6.15 & 1200 & 0.30 & 0.14 & +: \\
\hline
\end{tabular}

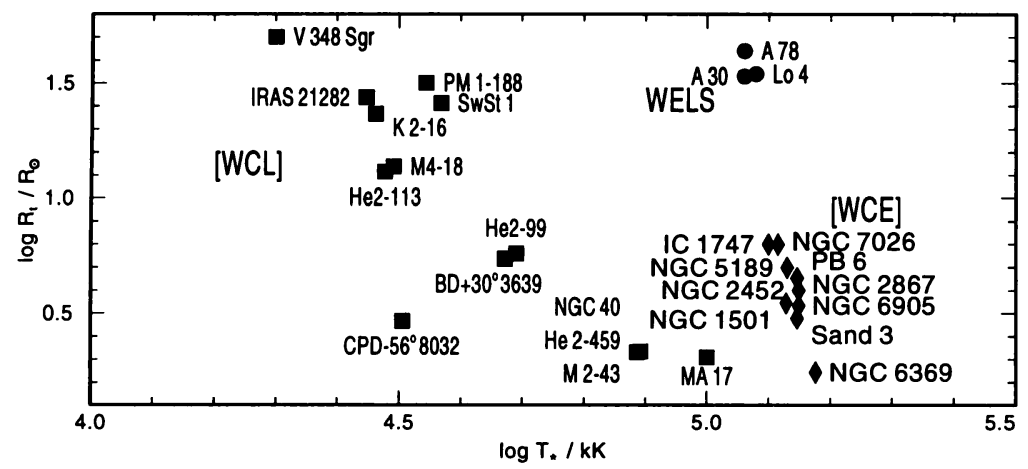

stars (Dreizler et al. 1995), the results presented here give constraints concerning the postAGB stages of low- and intermediate mass stars. Unfortunately detailed evolutionary tracks are not available so far but, hopefully, in the near future a detailed comparision between the analyses and the predictions of evolutionary calculations become practicable.

References

Hamann W-R., Leuenhagen U., Koesterke L., Wessolowski U. 1992, A\&A 255, 200

Hamann W.-R., Koesterke L., Wessolowski U. 1995, A\&A 299, 151

van der Hucht K.A., Conti P.S., Lundström I., Stenholm B. 181, Space Sci. Rev. 28, 227

Koesterke L., Hamann W.-R. 1996, A\&A in press

Leuenhagen U., Hamann W.-R 1996, A\&A 312, 167

Dreizler S., Werner K., Heber U. 1995, in "White Dwarfs", Lecture Notes in Physics, D. Koester \& K. Werner (eds), Springer-

Verlag, p. 160 